Article

\title{
An Exposed-Core Grapefruit Fibers Based Surface Plasmon Resonance Sensor
}

\author{
Xianchao Yang, Ying Lu *, Mintuo Wang and Jianquan Yao \\ College of Precision Instrument and Opto-Electronics Engineering, Key Laboratory of \\ Opto-electronics Information Technology, Ministry of Education, Tianjin University, \\ Tianjin 300072, China; E-Mails: yangxianchao@tju.edu.cn (X.Y.); wo3336903@126.com (M.W.); \\ jqyao@tju.edu.cn (J.Y.) \\ * Author to whom correspondence should be addressed; E-Mail: luying@tju.edu.cn; \\ Tel./Fax: +86-22-2740-6436.
}

Academic Editor: Vittorio M.N. Passaro

Received: 12 June 2015 / Accepted: 7 June 2015 / Published: 14 July 2015

\begin{abstract}
To solve the problem of air hole coating and analyte filling in microstructured optical fiber-based surface plasmon resonance (SPR) sensors, we designed an exposed-core grapefruit fiber (EC-GFs)-based SPR sensor. The exposed section of the EC-GF is coated with a SPR, supporting thin silver film, which can sense the analyte in the external environment. The asymmetrically coated fiber can support two separate resonance peaks ( $x$ - and $y$-polarized peaks) with orthogonal polarizations and $x$-polarized peak, providing a much higher peak loss than $y$-polarized, also the $x$-polarized peak has higher wavelength and amplitude sensitivities. A large analyte refractive index (RI) range from 1.33 to 1.42 is calculated to investigate the sensing performance of the sensor, and an extremely high wavelength sensitivity of $13,500 \mathrm{~nm} /$ refractive index unit (RIU) is obtained. The silver layer thickness, which may affect the sensing performance, is also discussed. This work can provide a reference for developing a high sensitivity, real-time, fast-response, and distributed SPR RI sensor.
\end{abstract}

Keywords: exposed-core grapefruit fibers; surface plasmon resonance; $x$ - and $y$-polarized; silver layer thickness 


\section{Introduction}

Propagating at the metal/dielectric interface, surface plasmons are extremely sensitive to changes in the refractive index of the dielectric [1]. Its high sensitivity to the refractive index (RI) variations of the dielectric adjacent to a metal shows great potential in chemical, biomedical, and industrial sensing [2]. Some surface plasmon resonance (SPR) configurations, such as the Kretschmann-Raether prism, can realize extremely high sensitivity, but present a number of disadvantages, such as costly integration, limited mechanical reliability, and difficulties in mass production [3]. Recently, optical fiber-based sensors have become more and more attractive because of their miniaturization, electromagnetic immunity, high sensitivity, and remote sensing capabilities. In most of the fiber-based SPR sensors, cladding is partially or completely stripped from the fiber and the exposed part is coated with a metal layer and then exposed to an analyte [2], which is very difficult and time-consuming. Additionally, the phase matching condition between the core mode and the plasmon mode is not easily achieved, because the fused silica material RI (about 1.45) is usually higher than bordering analyte (about 1.33). Theoretically, phase matching requires equating the propagation constants of the two modes, implying that the effective refractive indices of the two modes have to be similar. The effective RI of a core-guided mode is similar to the background silica, and the effective RI of a plasmonic mode is close to the bordering analyte [4].

The appearance of photonic crystal fibers (PCFs) is a breakthrough in fiber fiber-optic technology, leading to unprecedented properties that overcome many limitations [1]. In contrast with traditional optical fibers, PCFs have several geometric parameters that can be manipulated for larger greater flexibility of design [5]. The existence of air holes provide the possibility to insert analytes, then the mode effective RI could be tunable to manage the anticipated values, solving the phase matching problem. To realize SPR sensing, the air holes are selectively or completely coated with the metal layers, and then filled with analytes. One can also realize the SPR sensing by filling the silver nanowires [6,7]. However, we should note that either coating the metal films or filling analytes and silver nanowires into the air holes is difficult and time-consuming work, which makes it impossible for real-time, fast-response use. Some sensors, based on the D-shaped optical fibers, have been investigated $[2,8,9]$. The PCF is side-polished to form a flat plane and then coated with a metal film. Compared to the inside coating of the fiber holes, the outside coating is much easier. As the sensing region is exposed to the external environment, the sensors can realize fast-response and real-time sensing. However, the cladding air holes are too small and intensive, and it is very easy to destroy the cladding air holes when polished. Then Because of this, the plane will not be flat and the metal film will not be uniform, which may affect the sensing results. In [9], Tian et al. simulated an all-solid photonic fiber with D-shaped structure based surface plasmonic resonance sensor and a sensitivity of $7300 \mathrm{~nm} /$ refractive index unit (RIU) can be achieved. Recently, Luan et al. also reported a surface plasmon resonance sensor based on D-shaped microstructured optical fiber with hollow core, and identified the sensor sensitivity on wavelength, amplitude, and phase. The wavelength sensitivity they obtained was $2900 \mathrm{~nm} / \mathrm{RIU}$ when analyte RI changes from 1.33 to 1.34 [2].

In this paper, we design an exposed-core grapefruit fiber (EC-GF)-based SPR sensor to detect analyte RI. Two air hole claddings of the grapefruit fiber are polished and coated with a silver film to form an analyte channel. As the grapefruit fiber air holes are much larger $(80 \mu \mathrm{m})$ than the ordinary 
PCF (less than $2 \mu \mathrm{m}$ ), the operation can be much easier than with D-shaped optical fiber sensors, also grapefruit fibers have been produced by many manufacturers, which can be widely and practically used. Unlike the symmetrically coated fibers that support only one single resonance peak, the asymmetrically coated fiber can support two separate peaks, which are $x$-polarized and $y$-polarized peaks, respectively [10]. The $x$-polarized peak provides a much higher peak loss than $y$-polarized, and the $x$-polarized peak has higher wavelength and amplitude sensitivities. A large analyte RI range from 1.33 to 1.42 is calculated by the designed sensor, and an extremely high wavelength sensitivity of $13,500 \mathrm{~nm} / \mathrm{RIU}$ is obtained. The silver coating's thickness, which may affect the sensor's performance, is also discussed. This work can provide a reference for the implementation and application of EC-GF-based SPR sensors or other fibers-based SPR sensing.

\section{Structure Design and Simulated Modeling}

The cross-section of commercial grapefruit fiber is shown in Figure 1a. The schematic of the designed EC-GF-based SPR sensor is shown in Figure 1b. The thickness of the core struts are $c=2 \mu \mathrm{m}$. The diameters of the core and the air holes are $d_{c}=20 \mu \mathrm{m}$ and $d=80 \mu \mathrm{m}$, respectively. The exposed section of the fiber is coated with a $40 \mathrm{~nm}$ silver layer. Coating of the metal layers can be performed either with a chemical vapor deposition technique [11] or a wet chemistry deposition technique [12], which is much easier than inside coating of the fiber holes in operation. The refractive index of the EC-GF material is assumed to be 1.45 (fused silica), and the refractive index of the silver is given by the Handbook of Optics [13].

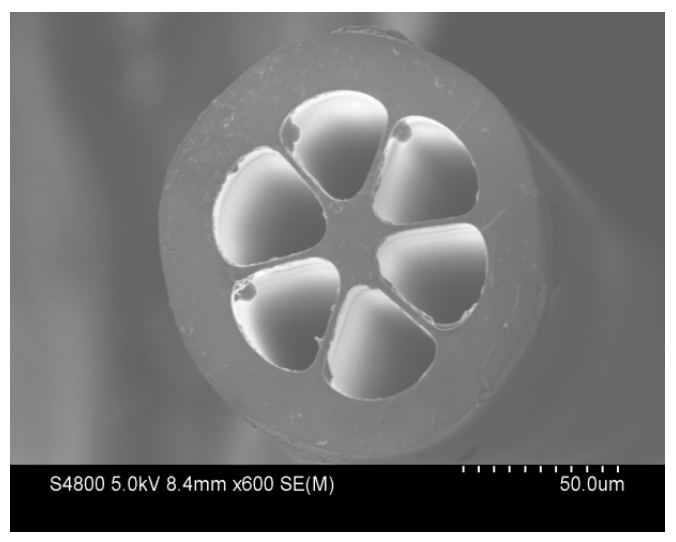

(a)

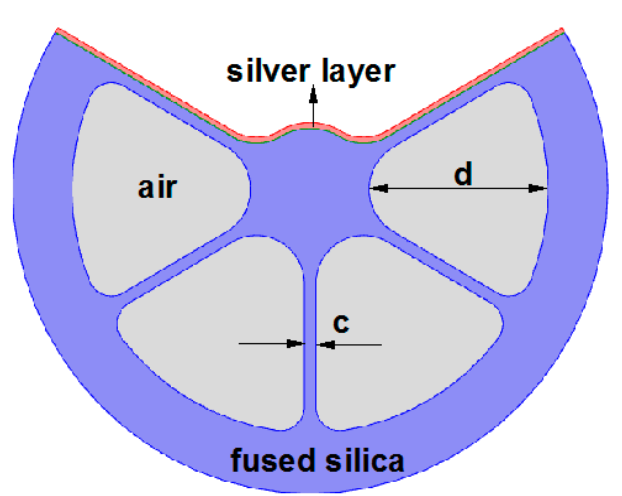

(b)

Figure 1. (a) Cross-section of the commercial grapefruit fiber; (b) Schematic of the designed EC-GF-based SPR sensor.

The electromagnetic mode of the sensor is solved by the finite element method (FEM) using COMSOL multiphysics software. Figure 2 shows the dispersion relations and electric field distributions of the core mode and plasmon mode when the RI of the liquid analyte is 1.42. The black solid curve represent the real parts of the effective RI of the $x$-polarized core mode and black dotted curve represent the real parts of the effective RI of $y$-polarized core mode. The red curve represents the plasmon mode. The blue solid curve and blue dotted curve represent the imaginary parts of the effective RI of $x$-polarized core mode and $y$-polarized core mode, respectively. Inset (a) represent 
electric field distributions of plasmon mode at $\lambda=1026 \mathrm{~nm}$; (b) represent $y$-polarized core mode at $\lambda=940 \mathrm{~nm}$; (c) represent $x$-polarized core mode at $\lambda=940 \mathrm{~nm}$; (d) represent $y$-polarized core mode at $\lambda=985 \mathrm{~nm}$ (phase matching point); and (e) represent $x$-polarized core mode at $\lambda=1026 \mathrm{~nm}$ (phase matching point).

Here, we use the Gaussian-like modes as the core modes, which are best suited for the excitation by standard Gaussian laser sources. As shown in Figure 2, the core modes exhibit strong birefringence, with one mode ( $y$-polarized mode see insets (b)) being polarized essentially parallel to the axis of symmetry and the other ( $x$-polarized mode see insets (c)) orthogonal to it [2]. Then, there will be two effective RI curves of the core modes, resulting in two intersections (dots (d) and (e)) with the plasmon mode and two resonance peaks for the same analyte index. We can see that the $x$-polarized resonance peak presents a much higher peak loss than $y$-polarized, also the $x$-polarized resonance peak has a higher coupling efficiency (insets (e) and (d)). When the phase matching is satisfied at a certain wavelength, the energy of a core mode is transferred to the plasmon mode and a resonant loss peak will be observed at this wavelength. The variation of the analyte RI will induce changes of the phase matching point between the core mode and the plasmon mode, thus leading to different loss spectra, which can be identified by measuring the peak wavelength shift or transmitted power change.

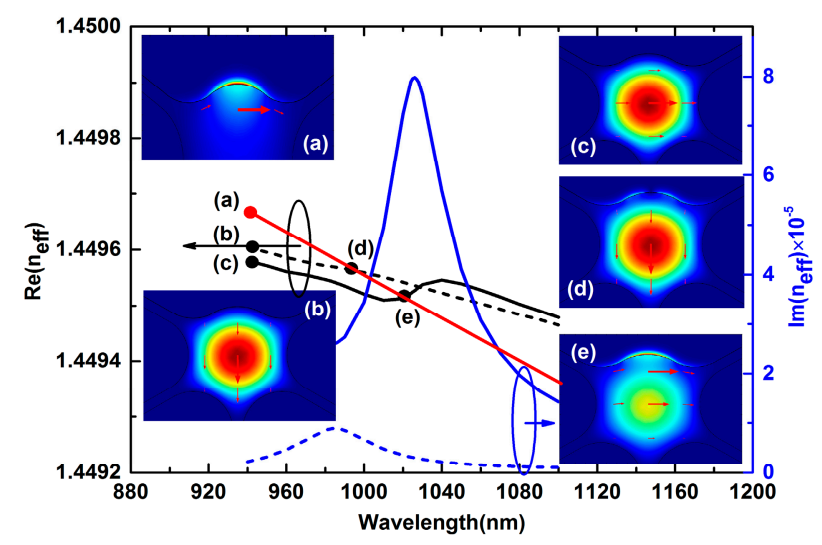

Figure 2. Dispersion relations and electric field distributions of core modes and the plasmon mode with analyte RI $n_{a}=1.42$. Insets (a) to (e) are electric field distributions of plasmon mode and core mode at different wavelengths.

\section{Results and Discussion}

\subsection{RI Sensitivity of the Sensor}

To investigate the sensing performance of the sensor, a large analyte RI range from 1.33 to 1.42 is calculated. Figure $3 \mathrm{a}$ shows the loss spectra of the $x$ - and $y$-polarized peaks with analyte RI 1.33 and 1.34. The confinement loss is defined as:

$$
\alpha_{\text {loss }}(d B / m)=8.686 \cdot k_{0} \operatorname{Im}\left[n_{\text {eff }}\right]
$$

where $k_{0}=2 \pi / \lambda$ is the wavenumber with $\lambda$ in meters and $\operatorname{Im}\left(n_{\text {eff }}\right)$ is the imaginary part of the mode effective RI. We can see that the $x$-polarized resonance peak presents a much higher peak loss than $y$-polarized, as the $x$-polarized resonance peak has a higher coupling efficiency. When the analyte RI 
changes from 1.33 to 1.34 , both the $x$ - and $y$-polarized peaks all shift to the longer wavelength, but the $x$-polarized resonance peak has a larger shift $(20 \mathrm{~nm})$ than the $y$-polarized $(19 \mathrm{~nm})$. The wavelength sensitivity is defined as:

$$
S_{\lambda}(n m / R I U)=\frac{\partial \lambda_{p e a k}}{\partial n_{a}}
$$

where $\lambda_{\text {peak }}$ is the resonance wavelength and $n_{a}$ is the analyte RI. Then the $x$-polarized resonance peak has a higher wavelength sensitivity $(2000 \mathrm{~nm} / \mathrm{RIU})$ than $y$-polarized $(1900 \mathrm{~nm} / \mathrm{R})$. If the spectral variation of $1 \mathrm{~nm}$ can be accurately detected by the spectrograph, then the sensor's detection resolution is $R=1 / S_{\lambda}$. The resolution of $x$ - and $y$-polarized peaks are $5 \times 10^{-4}$ RIU and $5.26 \times 10^{-4}$ RIU, respectively.

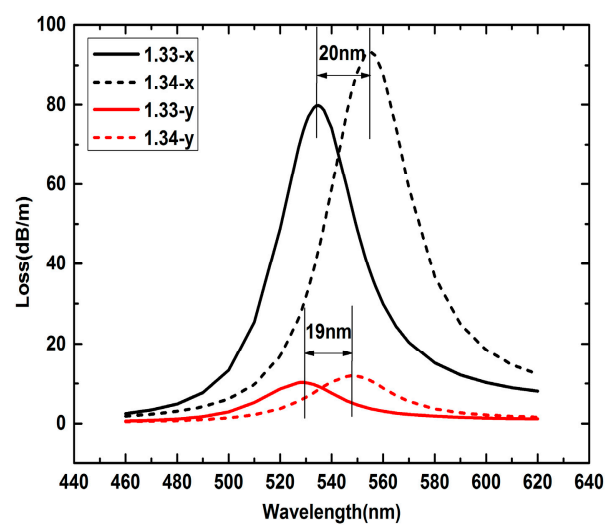

(a)

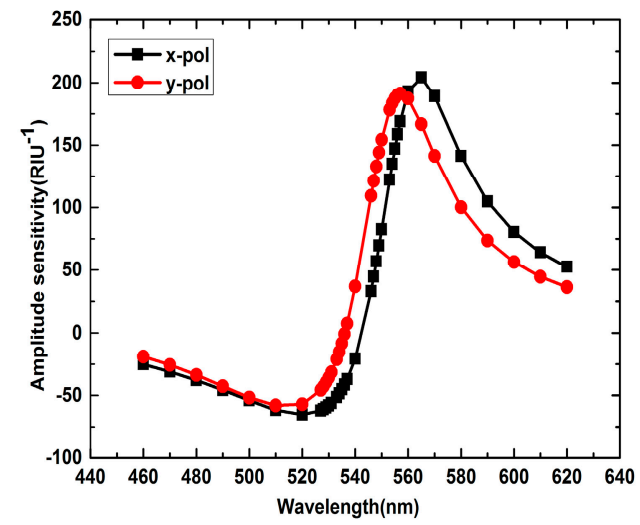

(b)

Figure 3. (a) Loss spectra of $x$ - and $y$-polarized peaks with analyte RI 1.33 and 1.34;

(b) Amplitude sensitivity of $x$ - and $y$-polarized core modes with analyte RI changes from 1.33 to 1.34 .

Another frequently used detection method is known as the power detection. Assume that the wavelength of the light is $\lambda$, and the transmission length is L, then the amplitude sensitivity can be defined as:

$$
S\left(R I U^{-1}\right)=\frac{1}{\alpha\left(\lambda, n_{a}\right)} \frac{\partial \alpha\left(\lambda, n_{a}\right)}{\partial n_{a}}
$$

Figure $3 \mathrm{~b}$ shows the amplitude sensitivity of the $x$ - and $y$-polarized core modes with analyte RI changes from 1.33 to 1.34 . From the picture we can see that the $x$-polarized resonance peak has a higher amplitude sensitivity $\left(204 \mathrm{RIU}^{-1}\right)$ than the $y$-polarized $\left(191 \mathrm{RIU}^{-1}\right)$. This is because the $x$-polarized core mode has a larger effective sensing region and stronger mode coupling than the $y$-polarized.

With the analyte RI increasing, as shown in Figure 4a, $x$ - and $y$-polarized peak losses all increases, but the $x$-polarized peak loss increases much more quickly than the $y$-polarized, resulting in the gap between the $x$-polarized peak loss and the $y$-polarized getting larger and larger. From Figure $4 \mathrm{~b}$ we can see that both the $x$ - and $y$-polarized peaks show higher sensitivity for the high analyte RI change than the low. For example, the $x$-polarized wavelength sensitivity is $13,500 \mathrm{~nm} / \mathrm{RIU}$ when analyte RI changes from 1.41 to 1.42 , which is much higher than $2000 \mathrm{~nm} / \mathrm{RIU}$ when analyte RI changes from 1.33 to 1.34 . 
The reason is that when the analyte RI increasing from 1.33 to 1.42 , the effective RI of the plasmonic mode is getting more and more close to the effective RI of the core-guided mode (1.45), then the mode coupling will be enhanced, leading to a larger peak loss and higher sensitivity. Considering the wavelength sensitivity and the loss peak amplitude, the $x$-polarized resonance peak is more suitable for $\mathrm{RI}$ sensing. The detailed wavelength sensitivities of $x$-polarized peaks when analyte RI increasing from 1.33 to 1.42 is $2000 \mathrm{~nm} / \mathrm{RIU}, 2400 \mathrm{~nm} / \mathrm{RIU}, 2800 \mathrm{~nm} / \mathrm{RIU}, 3300 \mathrm{~nm} / \mathrm{RIU}, 4100 \mathrm{~nm} / \mathrm{RIU}, 5200 \mathrm{~nm} / \mathrm{RIU}$, $6700 \mathrm{~nm} / \mathrm{RIU}, 9100 \mathrm{~nm} / \mathrm{RIU}$, and 13,500 nm/RIU, respectively. The highest wavelength sensitivity is $13,500 \mathrm{~nm} / \mathrm{RIU}$ and the minimum resolution is $7.41 \times 10^{-5} \mathrm{RIU}$ when the spectrograph resolution is $1 \mathrm{~nm}$, which is higher than other exposed-core fiber based SPR sensors $[9,10]$.

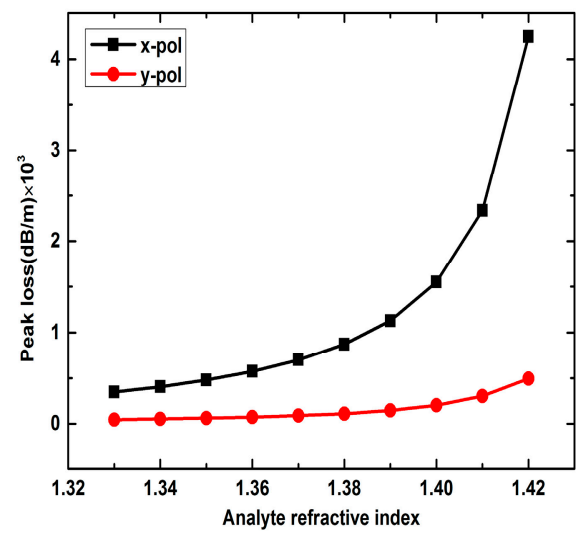

(a)

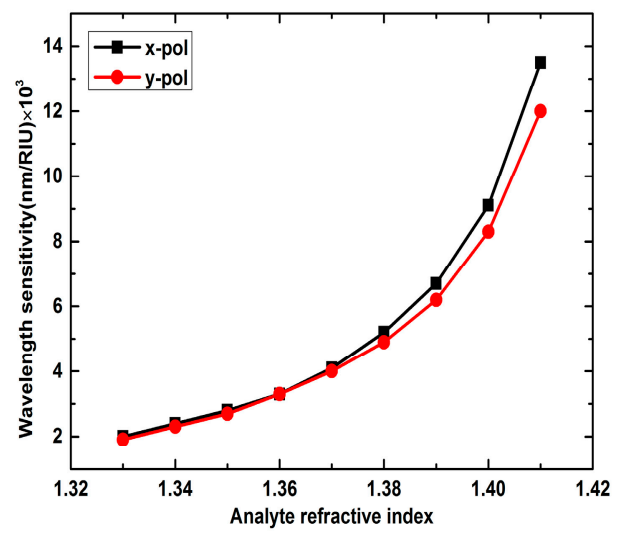

(b)

Figure 4. (a) $X$ - and $y$-polarized peak losses with analyte RI changes from 1.33 to 1.42 ;

(b) Wavelength sensitivity of $x$ - and $y$-polarized peaks with analyte RI changes from 1.33 to 1.42 .

In the fiber fabrication process, we can use higher RI background materials, such as polymer (PMMA $\mathrm{n} \approx 1.5$ ) [14], lead silicate (Schott F2, $\mathrm{n} \approx 1.62$ ) [15], and bismuth $(\mathrm{n} \approx 2.09)$ [16] to increase the upper detection limit, which can be used to detect some high RI organic chemical liquid analytes like benzene, nitrobenzene and phenylamine [10]. Additionally, we can use lower RI background materials or lower the RI of the core mode by inserting a central air hole $[2,4,17]$ to improve the sensitivity when detecting the low RI liquid analytes.

\subsection{Influence of Silver Layer Thickness on Sensitivity}

Surface plasmonic waves are very sensitive to the thickness of the silver layer. As shown in Figure 5a, the loss spectra of the $x$ - and $y$-polarized peaks vary considerably with the silver layer thickness changes from $30 \mathrm{~nm}$ to $50 \mathrm{~nm}$ when analyte RI is 1.33 . Generally, $x$ - and $y$-polarized peaks all shift to the longer wavelength as the silver layer becomes thicker and the peak losses decrease gradually. As the silver layer thickness continues to increase, from Figure $5 \mathrm{~b}$ we can see that the decrease of $x$ - and $y$-polarized peak losses become slower. Thus, when performing experiments, we can tune the peak loss to a desired value by adjusting the silver layer thickness. 


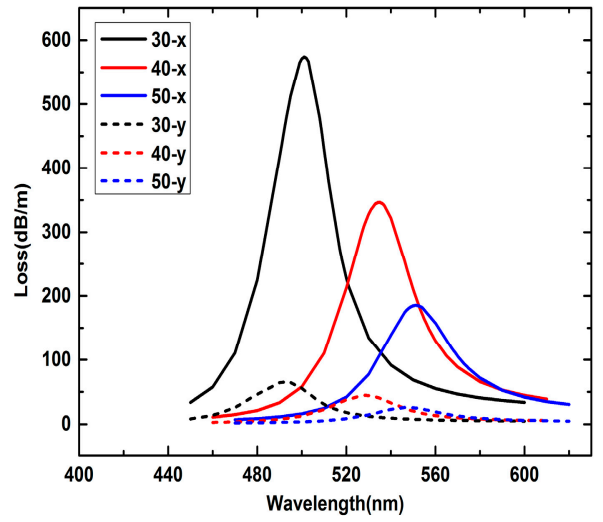

(a)

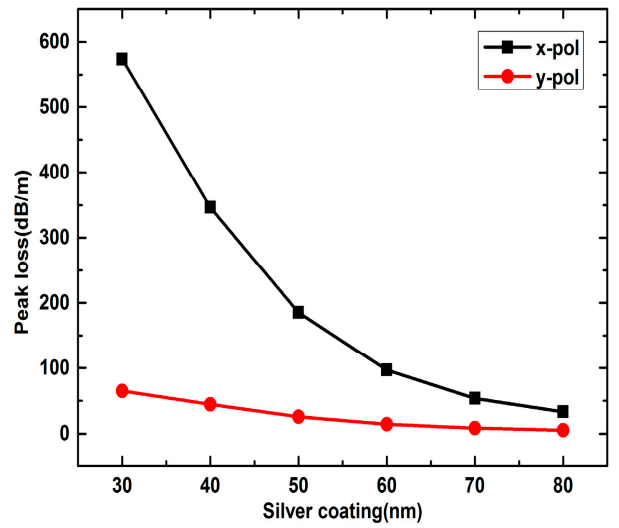

(b)

Figure 5. (a) Loss spectra of $x$ - and $y$-polarized peaks with silver layer thicknesses $30 \mathrm{~nm}$, $40 \mathrm{~nm}$ and $50 \mathrm{~nm}$ when analyte $\mathrm{RI}$ is 1.33 ; (b) $x$ - and $y$-polarized peak losses with silver layer thicknesses changes from $30 \mathrm{~nm}$ to $80 \mathrm{~nm}$ when analyte RI is 1.33 .

Figure 6a shows the wavelength sensitivity of the $x$ - and $y$-polarized peaks with different silver layer thicknesses when analyte RI is 1.33 . When the silver layer thickness changes from $30 \mathrm{~nm}$ to $80 \mathrm{~nm}$, the wavelength sensitivity of $x$ - and $y$-polarized peaks all go through a circle of rise and fall, which increase with the increases of silver layer thickness at first. When the silver layer is $70 \mathrm{~nm}$, the sensor has the maximum wavelength sensitivity of $2200 \mathrm{~nm} / \mathrm{RIU}$ and then it tends to decrease with the continued increase. The $x$-polarized peak always has a higher wavelength sensitivity than the $y$-polarized when the silver layer thickness is below $70 \mathrm{~nm}$, but then they have the same wavelength sensitivity when the thickness is more than $70 \mathrm{~nm}$. Contrary to the wavelength sensitivity, the amplitude sensitivity of the $x$ - and $y$-polarized peaks will always reduce with the silver layer thickness increase, as shown in Figure $6 \mathrm{~b}$, and the reduction rate is approximately linear.

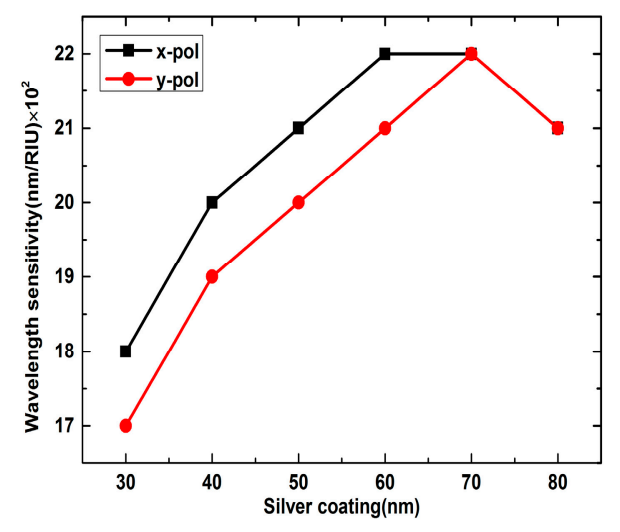

(a)

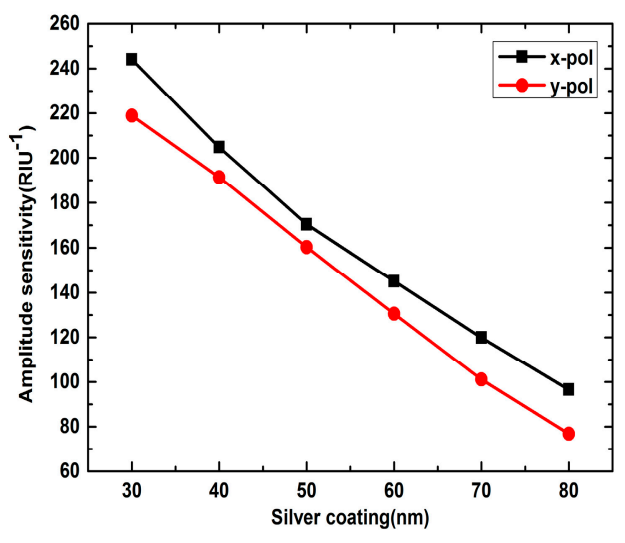

(b)

Figure 6. (a) Wavelength sensitivity of $x$ - and $y$-polarized peaks with silver layer thicknesses change from $30 \mathrm{~nm}$ to $80 \mathrm{~nm}$; (b) Amplitude sensitivity of $x$ - and $y$-polarized peaks with silver layer thicknesses change from $30 \mathrm{~nm}$ to $80 \mathrm{~nm}$

In conclusion, the thickness of the silver layer has a great influence on the sensor's performance. The reason is that the core mode of the fiber has a limited penetration depth. When the silver layer 
thickness increases to a value significantly larger than the penetration depth, the fiber core mode becomes effectively screened from the plasmon and the coupling between the core mode and the plasmon will weaken, resulting in a low coupling efficiency, and as a consequence, low sensitivity, and peak loss. Considering the peak loss amplitude and sensitivity, $40 \mathrm{~nm}$ is the optimized silver layer thickness for the sensor.

\section{Conclusions}

In this paper, we have analyzed an exposed-core grapefruit fiber based SPR sensor with a large analyte RI range from 1.33 to 1.42 and different silver layer thicknesses, from 30 to $80 \mathrm{~nm}$, through the finite element method using COMSOL Multiphysics software. Numerical results show that the asymmetrically coated fiber can support two orthogonal resonance peaks ( $x$ - and $y$-polarized peaks) and the $x$-polarised peak has a higher peak loss and higher sensitivity. Two polarized peaks all shift to the longer wavelength and exhibit increasing peak losses as the analyte RI increasing. The sensor shows a higher sensitivity for the high analyte RI changes than the low and the extremely high wavelength sensitivity $13,500 \mathrm{~nm} / \mathrm{RIU}$ is obtained. The silver layer thickness has a great influence on the sensor's performance. When the silver film becomes too thick, the wavelength and amplitude sensitivity all decreased. Thus, when performing experiments, we can tune the peak loss to a desired value by adjusting the silver layer thickness. The designed sensor is based on grapefruit fibers, which have larger air holes than common PCFs and are produced by many manufacturers. It can realize high sensitivity and sensing liquid analytes in the external environment, which is promising to develop a high sensitivity, real-time, fast-response and distributed SPR RI sensor.

\section{Acknowledgments}

This work was supported by the National Basic Research Program of China. (973 Program) (Grant number: 2010CB327801).

\section{Author Contributions}

Xianchao Yang designed the sensor architecture, carried out the simulations and drafted the manuscript. Ying Lu, Mintuo Wang and Jianquan Yao reviewed the paper and made a critical revision of the manuscript. All authors read and approved the final manuscript.

\section{Conflicts of Interest}

The authors declare no conflict of interest.

\section{References}

1. Barnes, W.L.; Dereux, A.; Ebbesen, T.W. Surface plasmon subwavelength optics. Nature 2003, 424, 824-830.

2. Luan, N.; Wang, R.; Lv, W.; Yao, J. Surface plasmon resonance sensor based on D-shaped microstructured optical fiber with hollow core. Opt. Express 2015, 23, 8576-8582. 
3. Lam, W.; Chu, L.; Wong, C.; Zhang, Y. A surface plasmon resonance system for the measurement of glucose in aqueous solution. Sens. Actuators B Chem. 2005, 105, 138-143.

4. Yu, X.; Zhang, Y.; Pan, S.; Shum, P.; Yan, M.; Leviatan, Y.; Li, C. A selectively coated photonic crystal fiber based surface plasmon resonance sensor. J. Opt. 2010, 12, 015005.

5. Wang, R.; Yao, J.; Miao, Y.; Lu, Y.; Xu, D.; Luan, N.; Musideke, M.; Duan, L.; Hao, C. A Reflective photonic crystal fiber temperature sensor probe based on infiltration with liquid mixtures. Sensors 2013, 13, 7916-7925.

6. Peng, Y.; Hou, J.; Zhang, Y.; Huang, Z.; Xiao, R.; Lu, Q. Temperat ure sensing using the bandgap-like effect in a selectively liquid-filled photonic crystal fiber. Opt. Lett. 2013, 38, $263-265$.

7. Lu, Y.; Wang, M.; Hao, C.; Zhao, Z.; Yao, J. Temperature sensing using photonic crystal fiber filled with silver nanowires and liquid. Photon. J. 2014, 6, 6801307.

8. Tan, Z.; Hao, X.; Shao, Y.; Chen, Y.; Li, X.; Fan, P. Phase modulation and structural effects in a D-shaped all-solid photonic crystal fiber surface plasmon resonance sensor. Opt. Express 2014, 22, 15049-15063.

9. Tian, M.; Lu, P.; Chen, L.; Lv, C.; Liu, D. All-solid D-shaped photonic fiber sensor based on surface plasmon resonance. Opt. Commun. 2012, 285, 1550-1554.

10. Luan, N.; Wang, R.; Lv, W.; Yao, J. Surface plasmon resonance sensor based on exposed-core microstructured optical fibres. Electron. Lett. 2015, 51, 714-715.

11. Sazio, P.J.A.; Correa, A.A.; Finlayson, C.E.; Hayes, J.R.; Scheidemantel, T.J.; Baril, N.F.; Jackson, B.R.; Won, D.J.; Zhang, F.; Margine, E.R.; et al. Microstructured optical fibers as high-pressure microfluidic reactors. Science 2006, 311, 1583-1586.

12. Harrington, J.A. A review of IR transmitting, hollow wave-guides. Fiber Integr. Opt. 2000, 19, 211-227.

13. Edward, D.P. Handbook of Optical Constants of Solids; Academic Press: Boston, MA, USA, 1985.

14. Cox, F.M.; Lwin, R.; Large, M.C.J.; Cordeiro, C.M.B. Opening up optical fibres. Opt. Express 2007, 15, 11843-11848.

15. Warren-Smith, S.C.; Ebendorff-Heidepriem, H.; Foo, T.C.; Moore, R.; Davis, C.; Monro, T.M. Exposed-core microstructured optical fibers for real-time fluorescence sensing. Opt. Express 2009, 17, 18533-18542.

16. Warren-Smith, S.C.; Afshar, S.; Monro, T.M. Theoretical study of liquid-immersed exposed-core microstructured optical fibers for sensing. Opt. Express 2008, 16, 9034-9045.

17. Peng, Y.; Hou, J.; Huang, Z.; Lu, Q. Temperature sensor based on surface plasmon resonance within selectively coated photonic crystal fiber. Appl. Opt. 2012, 51, 6361-6367.

(C) 2015 by the authors; licensee MDPI, Basel, Switzerland. This article is an open access article distributed under the terms and conditions of the Creative Commons Attribution license (http://creativecommons.org/licenses/by/4.0/). 